ISSN 1997-5902

\title{
Impact de la demande en chlore et de la chloration sur la désinfection des eaux de puits des quartiers de Lomé : cas des quartiers de Démakpoé et d'Agbalépédogan (Togo).
}

\author{
TAMPO L., AYAH M., KODOM T., TCHAKALA I., BOGUIDO P., BAWA L., DJANEYE B. \\ Laboratoire de Chimie des Eaux (LCE), Faculté Des Sciences, Université de Lomé. \\ charlestampo@gmail.com
}

Original submitted in on $4^{\text {th }}$ December 2013. Published online at www.m.elewa.org on $31^{\text {st }}$ March 2014. http://dx.doi.org/10.4314/jab.v75i1.12

\section{RÉSUMÉ}

Objectif : Le présent travail a pour but d'évaluer l'efficacité du chlore sur la désinfection des eaux de puits traditionnels. Il s'agit en outre d'apporter une contribution à la connaissance de la détermination de la demande en chlore pour la désinfection.

Méthodologie et résultats : Les méthodes décrites par l'Association Française de Normalisation (AFNOR) ont été utilisées. Selon nos résultats comparés aux normes de l'OMS la qualité physicochimique et bactériologique des eaux des puits est détériorée du fait du niveau d'assainissement et d'hygiène public dans ces quartiers. Les potentiels de consommation en chlore de ces eaux, montrent que les demandes en chlore sont considérables avec un pourcentage d'environ $90 \%$ des doses introduites. Les courbes de « Break Point » montrent une variation significative des demandes initiales et des valeurs de la dose de Break Point au cours du temps. Les chloramines éliminent $100 \%$ des coliformes totaux et réduisent au maximum le nombre de germes totaux à moins de 100 (norme OMS) dans ces eaux.

Conclusion et application des résultats : La demande en chlore varie selon les eaux des puits et au cours du temps. Le chlore est efficace sur l'ensemble des bactéries indicatrices et est capable de désinfecter ces eaux, les rendre potables et fournir un chlore résiduel si la demande en chlore est déterminée préalablement.

Mots clés : Eaux de puits, Qualité physicochimique et bactériologique, Désinfection au chlore, point de rupture.

Impact of the application for chlorine and chlorine disinfection of well water in districts of Lomé: case of districts of Démakpoé and Agbalépédogan (Togo).

ABSTRACT

Objective: The aim of this work was to evaluate the efficiency of chlorine disinfection of traditional wells water. On the other hand, this work is conducted to contribute to the knowledge of the chlorine demand determination for disinfection.

Methodology and results: Methods described by the French Standards Association were used. The results compared to WHO standards show that physicochemical and bacteriological quality of wells water has been deteriorated because of weak level of sanitation and public hygiene in these areas. Chlorine consumption of these waters shows that a high amount of chlorine corresponding about $90 \%$ of doses introduced has been 
reacted. The break-point curves show a significant variation of initial demand and values of break point dose over the time. When chloramines are used for disinfection, $100 \%$ of total coliforms were removed while maximum number of total bacteria was reduced less than 100 (WHO standard) in these waters.

Conclusion and application of results: Chlorine demand change according to wells water and over the time. The use of chlorine as disinfector was more efficient on indicators bacteria of these waters. Chlorine is able to disinfect the target waters leading them drinkable and provide residual chlorine if chlorine demand is preliminary determined.

Keys words: Well water, Physicochemical and bacteriological quality, Disinfection, Break Point.

\section{INTRODUCTION}

L'eau potable est un bien nécessaire mais limitée. Bien que l'eau soit la matière la plus abondante et commune de notre planète, seulement $0,003 \%$ du total est de l'eau douce disponible pour être utilisée dans les ménages ITC), 2007). Les villes du Togo en général et la ville de Lomé, la capitale en particulier, sont confrontées depuis quelques années à une importante pénurie d'eau (Bawa et al., 2008). Cette situation est commune dans la sous région ouest-africaine. Par exemple, au Mali, on assiste de façon répétitive à une dégradation de la qualité de l'eau distribuée aux populations de Bamako et ses environs en début d'hivernage. Ce phénomène a connu des points particulièrement critiques en 1993 et 1998. Ainsi, l'approvisionnement en eau potable des populations en quantité suffisante en toutes saisons demeure l'un des soucis majeurs des différents gouvernements (Hawa S., 2002). La pollution grandissante due aux activités humaines couplées aux changements climatiques augmente la vulnérabilité des eaux douces. Ainsi, plus d'efforts sont réalisés dans le traitement des eaux comme par exemple plus d'utilisation d'oxydants pour détruire aussi bien les micropolluants organiques et les micro-organismes. Le chlore et ses composés sont les agents désinfectants les plus utilisés à l'échelon mondial (FEPS, 2007 ; OMS, 2004). La chloration permet de détruire les bactéries et les virus présents dans l'eau potable et d'éviter la transmission de maladies hydriques dont les plus communes sont les gastro-entérites (OMS, 2004 ; REJSEK F, 2002). La destruction des bactéries a lieu par blocage de l'activité enzymatique (action biocide) par le chlore
$(\mathrm{HOCl})$ entraînant ainsi la mort de la cellule. Malgré son importance dans la désinfection, le chlore présente également des inconvénients tels que la formation des sous produits, les trihalométhanes, chlorophénols et organochlorés à toxicité chronique dont l'effet cumulatif peut également apparaitre sous l'aspect d'effets mutagènes voire cancérigènes. Ces sous produits entrainent aussi des mauvais goûts et odeurs du fait de leur présence dans certaines eaux de consommation après chloration (Catherine Juery, 2012; Guergazi S. et Achour S., 2011). Au Togo, en général, le traitement minimal des eaux envisagé par les populations est la désinfection (Bawa et al., 2008). Le réactif chimique le plus simple et le moins coûteux à mettre en place est le chlore sous sa forme liquide ou l'eau de javel 8 à $12^{\circ} \mathrm{Chl}$ dilué du millième au dix millième selon le potentiel de consommation en chlore de l'eau (Séby, 2006). La dose doit être déterminée expérimentalement pendant la période de chloration car une dose insuffisante reste inefficace sur certains germes alors qu'une dose trop élevée entraine un goût désagréable. II est donc nécessaire de déterminer en fonction de la qualité de l'eau à traiter, la quantité de chlore nécessaire pour limiter les inconvénients dans l'utilisation du chlore. Le but de cette étude est d'évaluer la qualité physicochimiques et bactériologiques des eaux de puits des quartiers Démakpoé et Agbalépédogan à Lomé et déterminer la dose et les formes de chlore (chlore combiné ou chlore libre) efficaces sur les bactéries indicatrices de contamination. 
MATÉRIEL ET MÉTHODES D'ANALYSE

Analyses physicochimiques : Les échantillons d'eau de onze puits et de onze forages ont été prélevés dans des bouteilles plastiques de $1,5 \mathrm{~L}$ puis acheminés au Laboratoire dans une glacière pour les analyses physicochimiques. La température, le $\mathrm{pH}$, la Conductivité et l'Oxygène dissous ont été mesurés in situ. Les autres paramètres physicochimiques ont été analysés au Laboratoire de Chimie des Eaux dans les $48 \mathrm{~h}$ selon les méthodes d'analyse de l'Association Française de Normalisation, AFNOR (AFNOR, 1986 ; Rodier J. et col. 2009). Le tableau 1 résume les méthodes et les équipements utilisés.

Tableau 1 : Matériel, méthodes et équipements utilisés

\begin{tabular}{lll}
\hline Paramètres & Méthodes & Équipements \\
\hline $\mathrm{pH}$ & Electrométrie & $\mathrm{pH}-$ mètre WTW pH330i \\
$\mathrm{O}_{2}$ dissous & Oxymétrie & Oxymètre WTW \\
Conductivité & Conductimétrie & Conductimètre WTW Cond 330i \\
Turbidité & Néphélométrie & Turbidimètre DRT100B, 20012 \\
$\mathrm{Ca}^{2+}, \mathrm{Mg}^{2+}, \mathrm{TH}$ & Complexométrie à l'EDTA & - \\
$\mathrm{Oxy} . \mathrm{KMnO}^{2}$ & Milieu acide et à chaud & - \\
$\mathrm{Chlore}$ & lodométrie/ Volumétrie & - \\
$\mathrm{Cl}$ & Volumétrique & - \\
$\mathrm{NH}^{-}, \mathrm{NO}_{3}^{-}, \mathrm{NO}_{2}, \mathrm{Fe}$ & Méthode spectrophotométrique. & Spectrophotomètre Digitron Elvi 675
\end{tabular}

$\mathrm{SO}_{4}{ }^{2+}$ et $\mathrm{Mn}^{2+}$

Préparation de la solution du chlore et détermination de la demande en chlore : Une solution commerciale d'hypochlorite de sodium (eau de Javel « la croix ») de concentration $12^{\circ}$ chlorimétrique (soit environ 38040 $\mathrm{mg} / \mathrm{L}$ ) est d'abord titrée au laboratoire pour vérifier le degré chlorimétrique puis diluée convenablement en fonction de la demande en chlore théorique de l'échantillon dont la gamme de concentration dans l'ensemble est comprise entre 0 et $30 \mathrm{mgCl}_{2} / \mathrm{L}$ (Bawa et al., 2008; Séby, 2006). Cinq puits présentant des concentrations en ammonium $>0,05 \mathrm{mg} / \mathrm{L}$ ont été retenus pour la détermination de la demande en chlore de leur eau. La demande en chlore des eaux a été déterminée par la méthode au « break point ». Dans une série de 15 flacons contenant $125 \mathrm{~mL}$ d'échantillon, on ajoute des doses croissantes de la solution de chlore préparée après avoir déterminé la Dose Théorique du Break Point (DTBP), (DTBP $\left.=7,6 \times\left[\mathrm{NH}^{+}\right]\right)$qui permet de situer approximativement le point de rupture. Après un temps de contact de deux heures conservé dans un milieu sombre, on dose le chlore résiduel dans chaque flacon puis on trace la courbe "dose de chlore résiduel en fonction de la dose de chlore introduite ». La demande en chlore (quantité de chlore à introduire pour avoir un chlore résiduel suffisant pour la désinfection) des cinq puits a été déterminée pendant trois campagnes dont deux en saison sèche et une en saison des pluies.
Chloration des échantillons : Les eaux de trois puits ont fait l'objet de chloration en vue d'évaluer l'effet de la dose ou les différentes formes de chlore (chlore combiné c'est-à-dire les chloramines et chlore libre soit l'acide hypochloreux ( $\mathrm{HOCl}$ ) ou l'ion hypochlorite ( $\mathrm{ClO}-)$ ) sur la désinfection. Pour chaque puits nous avons déterminé à travers une courbe de break point la dose de chlore qui correspond à la formation de la moitié du maximum des chloramines, la dose qui correspond à la formation du maximum de chloramines, la dose du break point et une dose de $25 \%$ au-delà de la dose du break point. Ces différentes doses ont été respectivement introduites dans les flacons F1, F2, F3 et F4 contenant chacun $125 \mathrm{~mL}$ de l'échantillon d'eau de puits. Après un temps de contact de deux heures nous effectuons la mise en culture.

Analyses microbiologiques : En plus du flacon témoin F0 sans chlore, on fait la mise en culture des prélèvements des quatre flacons précités dans des milieux de culture et dans des conditions requises selon AFNOR. Les analyses microbiologiques des échantillons sont réalisées selon la méthode décrite par l'Association Française de Normalisation et les germes sont recherchés par la méthode du nombre le plus probable (NPP). Le tableau 2 résume les méthodes d'analyse microbiologiques utilisées (AFNOR, 1986 ; Rodier J. et col. 2009). 
Tableau 2 : Méthodes d'analyses microbiologiques

\begin{tabular}{llll}
\hline Germes & Références & Milieux de culture & Conditions de culture \\
\hline Germes totaux & NF V 08- 051, 1999 & Plate Count Agar (PCA) & $30^{\circ} \mathrm{C} ; 24$ à 48h \\
Coliformes totaux & NF 050, 1992 & VRBL & $30^{\circ} \mathrm{C} ; 24$ à 48h \\
Coliformes thermotolérants & NF V08-01,1992 & VRBL & $44^{\circ} \mathrm{C} ; 24$ à 48h \\
Streptocoques fécaux & NFV08-05,1999 & Rote et Lisky & $37^{\circ} \mathrm{C} ; 24$ à 48h \\
anaérobies sulfito réducteurs & XP V08-0 1,1996 & TSN & $44^{\circ} \mathrm{C} ; 24$ à 48h \\
\hline
\end{tabular}

Corrélation entre la qualité physicochimique de l'eau et la demande en chlore : Le logiciel R (R STUDIO) nous a servi pour établir la matrice de corrélation de

\section{RÉSULTATS ET DISCUSSIONS}

Qualité physicochimique et bactériologique des eaux de puits: Quelques paramètres physicochimiques permettant de caractériser les eaux de 11 forages et de 11 puits ont été mesurés. Le tableau 3 montre une variation du $\mathrm{pH}$ de 5,1 à 7,4 et de 5,6 à 6,4 respectivement dans les forages et dans les eaux des puits. Ces valeurs de $\mathrm{pH}$ dans les eaux des puits sont très proches de celles de BAWA et al. (2008) qui ont obtenu des valeurs inférieures à 6,5 dans les eaux des puits des quartiers périurbains de Lomé. Elles sont par ailleurs différentes de celles de DERWICH et al, (2010) qui ont obtenus des valeurs variant de 7,1 et 8,1 dans les eaux des nappes alluviales du haut Sebou. Cette différence serait due à la nature très différente des roches. L'oxydabilité au $\mathrm{KMnO}_{4}$, le fer total et le Manganèse pratiquement absents dans certains forages
Spearman des paramètres physicochimiques et de la demande en chlore.

montrent des valeurs non négligeables avec des maxima de $9,3 \mathrm{mgO}_{2} / \mathrm{L} ; 1,54 \mathrm{mg} / \mathrm{L}$ et $7,5 \mathrm{mg} / \mathrm{L}$ dans les eaux des puits respectivement pour le $\mathrm{KMnO}_{4}$, le manganèse et le fer total. L'Oxygène dissous est plus faible dans les eaux des puits avec pour valeur maximale $4,3 \mathrm{mgO}_{2} / \mathrm{L}$ contre $5,7 \mathrm{mgO}_{2} / \mathrm{L}$ dans les eaux des forages. Ces valeurs sont voisines de celles d'Ali AïT BOUGHROUS (2007) qui a trouvé des valeurs inférieures à $6 \mathrm{mg} / \mathrm{L}$ dans les eaux souterraines de Tafilalet et Marrakech (Ali AïT BOUGHROUS, 2007). Ces valeurs relativement faibles s'expliqueraient dans le cadre de notre étude par l'absence de contact eau-atmosphère et du renouvellement perpétuel de l'eau (Hahn H. J., 2006). La turbidité est acceptable en comparaison avec les normes OMS-UE. Le tableau 3 présente les résultats des paramètres physico-chimiques décrits ci-dessus.

Tableau 3 : Paramètres physicochimiques et éléments métalliques

\begin{tabular}{lcccc}
\hline Paramètres & Unité & $\begin{array}{c}\text { Concentration maximale } \\
\text { OMS }\left({ }^{*}\right)-\text { UE }\end{array}$ & Forages & Puits \\
\hline $\mathrm{pH}$ & - & $6,5-8,5$ & $5,1-7,4$ & $5,6-6,4$ \\
Température & ${ }^{\circ} \mathrm{C}$ & - & $28,5-29,5$ & $28,3-29,5$ \\
Turbidité & $\mathrm{NTU}$ & $25\left(^{*}\right)-10$ & $1-4,45$ & $1-13,7$ \\
$\mathrm{O}_{2}$ dissous & $\mathrm{mgO}_{2} \mathrm{~L}$ & - & $4,6-5,7$ & $2,6-4,3$ \\
$\mathrm{KMnO}_{4}$ & $\mathrm{mgO}_{2} / \mathrm{L}$ & 5 & $0-0,3$ & $0,5-9,3$ \\
$\mathrm{Mn}^{2+}$ & $\mathrm{mg} / \mathrm{L}$ & $0,5\left(^{*}\right)-0,05$ & $0-0$ & $0-1,54$ \\
Fer total & $\mathrm{mg} / \mathrm{L}$ & $0,3\left(^{*}\right)-0,2$ & $0-0,38$ & $0-7,50$ \\
\hline
\end{tabular}

Le tableau 4 présente les résultats des paramètres liés à la minéralisation. Les valeurs de la conductivité que nous avons trouvées en ce qui concerne les eaux des forages se rapprochent de celles de BRICHA et al., 2007 qui ont trouvés des valeurs variant de $562 \mu \mathrm{s} / \mathrm{cm}$ à $1493 \mu \mathrm{s} / \mathrm{cm}$ dans les eaux des puits de la nappe phréatique de M'nasra au Maroc. Les conductivités dans les eaux de puits de la présente étude, sont plus élevées par rapport aux normes OMS-UE. Ces dernières placent ces eaux dans les eaux fortement minéralisées comme reportèrent Taouil et al, 2013 qui ont trouvé des valeurs comprise entre 1250 et $1420 \mu \mathrm{s} / \mathrm{cm}$ dans les eaux des puits de Tyikomiyn du Maroc Oriental (Taouil et al., 2013). Les concentrations en $\mathrm{SO}_{4}{ }^{2-}, \mathrm{Cl}, \mathrm{HCO}_{3}{ }^{-}, \mathrm{Ca}^{2+}$ et $\mathrm{Mg}^{2+}$ avec des valeurs médianes respectives de 29,$7 ; 206,7$; 210,$35 ; 44,6$ et $56,64 \mathrm{mg} / \mathrm{L}$ dans les eaux des forages et de 60,$05 ; 385,4 ; 263,52 ; 109,6$ et $58,08 \mathrm{mg} / \mathrm{L}$ dans les eaux des puits indiquent une bonne qualité des eaux des 
forages et une qualité un peu détériorée des eaux des puits en comparaison avec les normes OMS-UE. Les concentrations en chlorure un peu élevées dans certains puits seraient liées à la nature des terrains traversés. Ces valeurs en chlorure sont tout de même voisines de celles trouvées par Wassila Hamdi, (2011) dans les eaux d'Ouargla en Algérie.

Tableau 4 : Minéralisation, dureté et ions majeurs

\begin{tabular}{lcccc}
\hline Paramètres & Unité & $\begin{array}{c}\text { Concentration maximale } \\
\text { OMS(*)-UE }\end{array}$ & Forages & Puits \\
\hline conductivité & $\mu \mathrm{s} / \mathrm{cm}$ & $400($ valeur guide $)$ & $545-1484$ & $554-4460$ \\
Salinité & $\mathrm{mg} / \mathrm{L}$ & 1500 & $390,1-1277,3$ & $396,61-3389,1$ \\
$\mathrm{Cl}^{-}$ & $\mathrm{mg} / \mathrm{L}$ & $250\left(^{*}\right)-200$ & $103,1-310,3$ & $110,1-660,7$ \\
$\mathrm{SO}_{4}{ }^{2-}$ & $\mathrm{mg} / \mathrm{L}$ & $400\left({ }^{*}\right)-250$ & $2,6-56,8$ & $12,2-107,9$ \\
$\mathrm{HCO}_{3}{ }^{-}$ & $\mathrm{mg} / \mathrm{L}$ & $>30$ & $24,2-396,5$ & $36,6-490,44$ \\
$\mathrm{TH}^{2 a^{2+}}$ & $\mathrm{mgCaCO} / \mathrm{L}$ & 150 & $90-520$ & $134-846$ \\
$\mathrm{Mg}^{2+}$ & $\mathrm{mg} / \mathrm{L}$ & 100 & $16,8-72,4$ & $24,8-194,4$ \\
\hline
\end{tabular}

Le tableau 5 montre la variation de la concentration en éléments azotés et phosphatés. Les concentrations en ces éléments indiquent des valeurs nulles ou tolérables dans les eaux des forages alors que ces concentrations dépassent en moyenne les normes OMS-UE dans les eaux des puits. Cette situation au niveau des eaux des puits peut être expliquée par le lessivage des fertilisants agricoles qui s'infiltrent dans ces puits très peu profonds situés à proximité des champs de maïs et de légumes. La concentration en ammonium et en nitrites élevée dans certains puits serait due à l'insuffisance d'Oxygène dissous qui entraine une Oxydation incomplète du $\mathrm{NH}_{4}{ }^{+}$ou une absence totale de son oxydation, les plus faibles valeurs en Oxygène dissous étant enregistrées dans les eaux de ces puits.

Tableau 5 : Composés minéraux azotés et phosphatés

\begin{tabular}{lcccc}
\hline Paramètres & Unité & $\begin{array}{c}\text { Concentration maximale } \\
\text { OMS }\left(^{*}\right)-\text { UE }\end{array}$ & Forages & Puits \\
\hline $\mathrm{NO}_{3}{ }^{-}$ & $\mathrm{mg} / \mathrm{L}$ & $50\left(^{*}\right)-25$ & $0,5-70,3$ & $0,79-661,1$ \\
$\mathrm{NO}_{2}{ }^{-}$ & $\mathrm{mg} / \mathrm{L}$ & $3,0\left(^{*}\right)-0,2$ & $0-0$ & $0-1,97$ \\
$\mathrm{NH}_{4}{ }^{+}$ & $\mathrm{mg} / \mathrm{L}$ & 0,5 & $0-0$ & $0-3,40$ \\
$\mathrm{PO}_{4}{ }^{-{ }^{-}}$ & $\mathrm{mg} / \mathrm{L}$ & - & $0-0$ & $0-1,3$ \\
\hline
\end{tabular}

La détermination de la demande en chlore, le $\mathrm{pH}$, le Fer, le Manganèse, la turbidité, l'Ammonium et l'Oxydabilité au $\mathrm{KMnO}_{4}$ des eaux de cinq puits a été effectuée pendant les trois campagnes avant les essais de chloration..

Tableau 6 : variation des paramètres mesurés au cours des trois campagnes pour les eaux de puits

\begin{tabular}{|c|c|c|c|c|c|}
\hline Paramètres & Unités & $\begin{array}{c}\text { Directives OMS }\left(^{*}\right)- \\
\text { UE }\end{array}$ & Camp1 & Camp2 & Camp3 \\
\hline $\mathrm{pH}$ & & $6.50-8.50$ & $5,6-6,05$ & $5,9-6,3$ & $5,5-6,2$ \\
\hline Turbidité & NT̄U & 5 & $7.8-13.7$ & $7-21,8$ & $5,6-30,5$ \\
\hline conductivité & $\mu \mathrm{s} / \mathrm{cm}$ & $400\left(^{*}\right)$ & $3770-550$ & $4018-560$ & $4460-580$ \\
\hline $\mathrm{NH}_{4}^{+}$ & $\mathrm{mg} / \mathrm{L}$ & 1.5 & $0,4-3,4$ & $0,75-12,8$ & $0,83-14,4$ \\
\hline Oxy. $\mathrm{KMnO}_{4}$ & $\mathrm{mgO}_{2} / \mathrm{L}$ & 5 & $0,8-9,3$ & $0,6-11,1$ & $0,7-15,8$ \\
\hline $\mathrm{Fe}$ & $\mathrm{mg} / \mathrm{L}$ & 0.3 & $0,09-7,5$ & $0,15-7,9$ & $0,1-6,9$ \\
\hline $\mathrm{Mn}$ & $\mathrm{mg} / \mathrm{L}$ & 0.4 & $0,49-1,54$ & $0,5-2,4$ & $0,36-3,2$ \\
\hline
\end{tabular}

Le tableau 6 indique les variations des paramètres physicochimiques mesurés au cours des trois campagnes
Le tableau 6 montre que tous les paramètres sont dynamiques dans les eaux des cinq puits au cours des 
trois campagnes. Le pH varie de 5,5 à 6,3 , valeurs en dessous de la norme OMS. Ces $\mathrm{pH}$ acides peuvent s'expliquer selon Meybeck et al. (1996) par la teneur un peu élevée de la matière organique exprimée par les valeurs de l'oxydabilité au $\mathrm{KMnO}_{4}$. La turbidité et la conductivité varient respectivement de 7 à 30,5 NTU et de 550 à $4460 \mu \mathrm{s} / \mathrm{cm}$, largement au dessus des normes requises. Au regard de la conductivité, ces eaux sont qualifiées de moyennement minéralisées à eaux salines. Les concentrations en ammonium et en matières oxydables $\left(\mathrm{KMnO}_{4}\right)$ qui influencent fortement la demande en chlore, avec un coefficient de corrélation de l'ordre de 0,91 (voire tableau 9) varient respectivement de 0,4 à $14,4 \mathrm{mg} / \mathrm{L}$ et de 0,6 à $15,8 \mathrm{mgO}_{2} / \mathrm{L}$. L'analyse des résultats indiquent que plus de $80 \%$ des valeurs mesurées sont au dessus des valeurs maximales admises par les normes OMS. Ces résultats sont comparables à ceux de Makoutode et al. (1999) qui ont travaillé sur la qualité et la gestion des puits du grand PoPo au Benin, localité se situant dans la même ceinture du bassin sédimentaire côtier du Togo et du Benin. Les teneurs en fer et en Manganèse qui influencent également la chloration ont connu une variation respectivement de 0,09 à $7,9 \mathrm{mg} / \mathrm{L}$ et de 0,36 à $3,2 \mathrm{mg} / \mathrm{L}$. L'analyse de ces valeurs montre que les concentrations en $\mathrm{Mn}^{2+}$ sont hors des normes OMS au cours des campagnes 1 et 2 correspondant à la saison sèche.

L'analyse bactériologique a donné des résultats qui sont indiqués dans le tableau 7. Ces résultats de la bactériologie indiquent que la qualité de ces eaux est hors normes OMS. Les germes totaux varient de 23 $\mathrm{Ufc} / \mathrm{mL}$ dans le puits $\mathrm{P} 11$ à $9500 \mathrm{Ufc} / \mathrm{mL}$ dans le puits $\mathrm{P} 1$. En outre, il est constaté la présence d'une contamination en coliformes totaux dans les trois puits et la présence des anaérobies sulfito-réducteurs dans les puits 10 et 11. Les trois puits sont donc impropres à la consommation et présente un risque sanitaire ou hygiénique très élevé vue le nombre de germes totaux présents dans ces eaux. Ces résultats sont comparables à ceux de Fambi Komlan, (2009) qui a trouvé des valeurs variant de 10 à 26000 $\mathrm{Ufc} / \mathrm{mL}$ de germes totaux, de 0 à $330 \mathrm{Ufc} / \mathrm{mL}$ de coliformes totaux et 0 à 2 a Ufc/mL d'naérobies sulfitoréducteurs au cours de deux campagnes dans les puits de la région centrale au Togo. L'ensemble des paramètres physicochimiques et bactériologiques mesurés montre que la qualité des eaux des puits est impropre à la consommation. Cette situation pourrait s'expliquer par le niveau faible de l'assainissement et de l'hygiène publique dans ces quartiers.

Tableau 7: Résultats des analyses bactériologiques

\begin{tabular}{lccccc}
\hline Germes & Unité & Directive OMS & P1 & P10 & P11 \\
\hline Germes totaux & Ufc/mL & 100 & 9500 & 310 & 23 \\
Coliformes totaux & Ufc/mL & 0 & 10 & 6 & 3 \\
Coliformes thermotolérants & Ufc/mL & 0 & 0 & 0 & 0 \\
Streptocoques fécaux & Ufc/mL & 0 & 0 & 0 & 0 \\
Anaérobies sulfito-réducteurs & $\mathrm{Ufc} / \mathrm{mL}$ & 0 & 0 & 2 & 1 \\
\hline
\end{tabular}

\section{Détermination de la demande en chlore au cours des} trois campagnes : Le tableau 8 montre la dose de break point (dose correspondant à la plus faible valeur du chlore résiduel), la demande immédiate (quantité de chlore consommé avant sa réaction avec l'Ammonium) ainsi que la demande en chlore (quantité de chlore à introduire pour avoir un chlore résiduel suffisant pour la désinfection) au cours des trois campagnes. Ces valeurs varient plus ou moins au cours des trois campagnes mais montrent des écarts plus importants entre la première campagne (saison sèche) et la troisième (saison pluvieuse). La demande immédiate, révélée par les courbes de break point, indique une variation significative des valeurs entre les campagnes. Cette demande initiale non nulle indique la présence de certains composés dans ces eaux qui consommeraient rapidement une partie du chlore avant sa réaction avec l'Ammonium. Ces résultats montrent que la quantité de chlore consommée varie non seulement d'un puits à un autre mais aussi dans le temps dans un même point d'eau. Cette variation dans le temps est plus remarquée entre la première campagne et la troisième où les doses au point de rupture passent respectivement d'une valeur médiane de $38,05 \mathrm{mg} / \mathrm{L}$ à $60,7 \mathrm{mg} / \mathrm{L}$ de chlore. Le tableau 9 présente la matrice de corrélation entre les paramètres physico-chimiques et la demande en chlore. La corrélation entre les paramètres de la demande en chlore et les variables physicochimiques montre que la demande en chlore est très fortement corrélée avec la concentration en Ammonium, en Nitrite, en Nitrates et à l'Oxydabilité au $\mathrm{KMnO}_{4}$ avec un coefficient de l'ordre de 0,95 . Elle est également corrélée avec la température, le 
calcium et la minéralisation avec un coefficient de l'ordre de 0,65 . La demande immédiate quant à elle est très fortement corrélée avec le fer et le manganèse et faiblement avec la température, le $\mathrm{pH}$ ainsi que l'Oxydabilité au $\mathrm{KMnO}_{4}$.

Tableau 8 : variations de la demande en chlore des puits au cours des trois campagnes.

\begin{tabular}{lllll}
\hline Paramètres & unités & Camp1 & Camp2 & Camp3 \\
\hline Demande en chlore & $\mathrm{mg} / \mathrm{L}$ & $6,7-67$ & $7,9-106$ & $8,1-115$ \\
Demande en chlore immédiate & $\mathrm{mg} / \mathrm{L}$ & $0,7-3$ & $0,25-5$ & $0,13-4,5$ \\
Break point & $\mathrm{mg} / \mathrm{L}$ & $5,1-63$ & $5,8-106$ & $6,4-115$ \\
\hline
\end{tabular}

La figure 1 montre les variations des courbes de break point de trois puits aucour des trois campagnes. Ces courbes indiquent une variation significative des demandes en chlore aucours du temps dans le puits P11 et moins significative dans les puits P2 et P6. Cela s'explique par la variation des concentrations en ions Ammonium et en matières oxydables dans les eaux des puits P11 alors qu'elles sont plus stables dans les eaux des puits $\mathrm{P} 2$ et $\mathrm{P} 6$ pendant les trois campagnes.

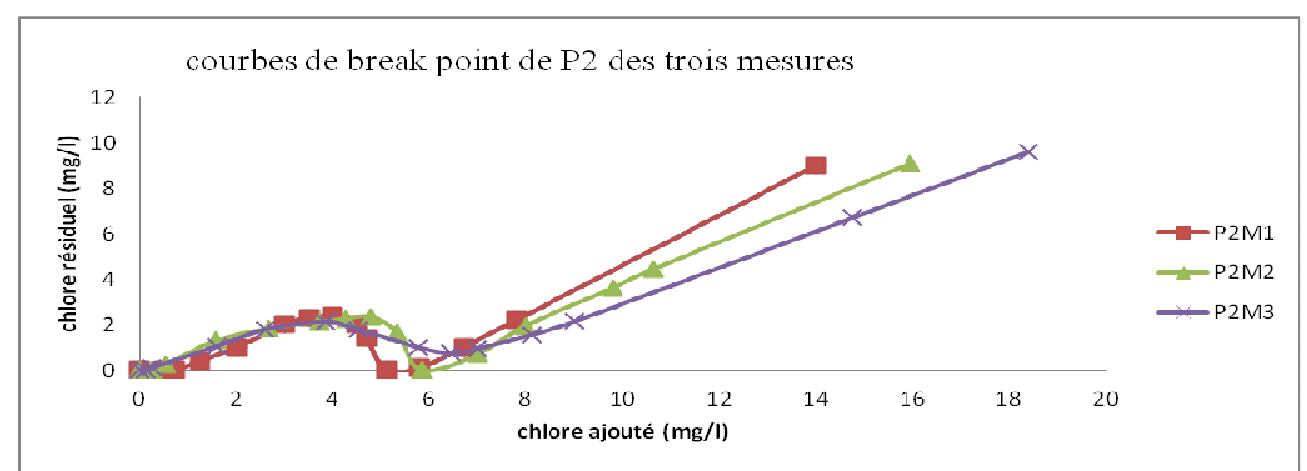

Figure 1 : courbes de break point des trois puits au cours des trois campagnes

Tableau 9 : Matrice de corrélation des paramètres physicochimiques et de la demande en chlore

\begin{tabular}{|c|c|c|c|c|c|c|c|c|c|c|c|c|c|c|c|}
\hline & Temp & $\mathrm{PH}$ & Sali & Turb & KMnO4 & $\mathrm{NH} 4$ & NO2 & NO3 & Fer & Mn & $\mathrm{Ca}$ & $\mathrm{Mg}$ & $\mathrm{DCl} 2$ & Di & $\mathrm{BP}$ \\
\hline Temp & 1,000 & & & & & & & & & & & & & & \\
\hline PH & 0,116 & 1,000 & & & & & & & & & & & & & \\
\hline Sali & $-0,024$ & $-0,902$ & 1,000 & & & & & & & & & & & & \\
\hline Turb & $-0,677$ & $-0,098$ & 0,232 & 1,000 & & & & & & & & & & & \\
\hline KMnO4 & 0,780 & $-0,325$ & 0,517 & $-0,188$ & 1,000 & & & & & & & & & & \\
\hline NH4 & 0,903 & $-0,180$ & 0,374 & $-0,442$ & 0,950 & 1,000 & & & & & & & & & \\
\hline NO2 & 0,736 & $-0,438$ & 0,626 & $-0,397$ & 0,894 & 0,937 & 1,000 & & & & & & & & \\
\hline NO3 & 0,670 & $-0,426$ & 0,620 & $-0,443$ & 0,794 & 0,876 & 0,981 & 1,000 & & & & & & & \\
\hline Fer & 0,579 & 0,308 & $-0,469$ & $-0,348$ & 0,297 & 0,288 & $-0,008$ & $-0,155$ & 1,000 & & & & & & \\
\hline Mn & 0,519 & 0,590 & $-0,727$ & $-0,402$ & 0,086 & 0,149 & $-0,176$ & $-0,283$ & 0,943 & 1,000 & & & & & \\
\hline $\mathrm{Ca}$ & 0,324 & $-0,838$ & 0,791 & $-0,396$ & 0,525 & 0,538 & 0,760 & 0,783 & $-0,216$ & $-0,434$ & 1,000 & & & & \\
\hline $\mathrm{Mg}$ & $-0,360$ & $-0,297$ & 0,408 & 0,890 & 0,198 & $-0,106$ & $-0,107$ & $-0,223$ & $-0,050$ & $-0,231$ & $-0,167$ & 1,000 & & & \\
\hline $\mathrm{DCl} 2$ & 0,695 & $-0,396$ & 0,641 & $-0,270$ & 0,911 & 0,931 & 0,987 & 0,963 & $-0,060$ & $-0,221$ & 0,683 & 0,001 & 1,000 & & \\
\hline Di & 0,558 & 0,410 & $-0,553$ & $-0,331$ & 0,242 & 0,246 & $-0,068$ & $-0,209$ & 0,994 & 0,970 & $-0,311$ & $-0,069$ & $-0,112$ & 1,000 & \\
\hline BP & 0,688 & $-0,407$ & 0,652 & $-0,252$ & 0,916 & 0,929 & 0,985 & 0,958 & $-0,058$ & $-0,225$ & 0,683 & 0,023 & 1,000 & $-0,111$ & 1,0 \\
\hline
\end{tabular}

Potentiel de Consommation en Chlore (P.C.Cl 2 ) et pourcentage du Chlore consommé : Le tableau 10 indique le potentiel de consommation en chlore (quantité de chlore consommée par une eau pour une dose introduite donnée) et le pourcentage de chlore consommé en considérant la dose introduite au point de rupture. Ces résultats indiquent que le P.C. $\mathrm{Cl}_{2}$ varie selon les puits et les campagnes avec des valeurs comprises entre 5,1 
$\mathrm{mgCl}_{2} / \mathrm{L}$ dans le puits $\mathrm{P} 1$ à la première campagne et $111,26 \mathrm{mgCl} / \mathrm{L}$ dans le puits $\mathrm{P} 10$ à la troisième campagne. Le pourcentage du chlore consommé est compris entre $85,14 \%$ dans le puits $\mathrm{P} 11$ à la première campagne et $100 \%$ dans le puits P2 à la première et deuxième campagne. Cette consommation est corrélable avec la qualité de l'eau de ces puits, la quantité et la nature des matières organiques. Au vue de l'ensemble des valeurs que nous avons trouvées, ces résultats sont comparables à ceux de Guergazi S. et Achour S., (2011) qui ont trouvé des pourcentages de consommation en chlore de 90,4 et 92,01 avec les eaux de deux barrages, Shayeb et al.(1998) qui ont obtenu des pourcentages de consommation de chlore de l'ordre de $90 \%$ sur une eau usée épurée au stade secondaire quelque soit la dose de chlore introduite de $1 \mathrm{mg} / \mathrm{L}$ à $10 \mathrm{mg} / \mathrm{L}$. La petite différence serait due à la nature des eaux, nos eaux étant des puits dont la qualité dépend de l'état d'assainissement des quartiers où nous avons fait le prélèvement.

Tableau 10 : Potentiel de Consommation en Chlore et pourcentage de consommation de chlore

\begin{tabular}{ccccccc} 
Puits & \multicolumn{3}{c}{ P.C.Cl $(\mathbf{m g C l} / \mathbf{l})$} & \multicolumn{3}{c}{$\%{\mathbf{~ d e ~} \mathbf{C l}_{2} \text { consommé }}$} \\
\cline { 2 - 7 } P1 & $\mathrm{C} 1$ & $\mathrm{C} 2$ & $\mathrm{C} 3$ & $\mathrm{C} 2$ & $\mathrm{C} 2$ & $\mathrm{C}$ \\
P2 & 9,7 & 11,06 & 17,32 & 89,8 & 92,32 & 95,58 \\
P6 & 5,1 & 5,84 & 5,67 & 100 & 100 & 88,18 \\
P10 & 11,93 & 10,47 & 11.12 & 90,17 & 86,74 & 87,01 \\
P11 & 54,03 & 98,4 & 111,26 & 85,76 & 92,83 & 96,74 \\
& 5,96 & 9,3 & 7,34 & 85,14 & 92,9 & 94,10
\end{tabular}

Inactivation des germes totaux et des coliformes totaux : Les nombres de germes après traitement au chlore sont présentés dans le tableau 11. Ces résultats montrent que le chlore a une action bactéricide complète sur les coliformes totaux et réduit fortement le nombre de germes totaux. Les courbes d'inactivation des germes (figure 2) montrent que les chloramines sont efficaces sur les coliformes totaux. En effet la première et la deuxième dose des courbes de break point ont généré au maximum des chloramines. Ces dernières ont éliminées complètement les coliformes totaux. Ces chloramines réduisent un grand nombre de germes totaux mais n'éliminent pas certains germes qui seront probablement des formes résistantes même au chlore libre puisque le nombre de germes totaux reste pratiquement constant après des doses au-delà du point de rupture. Ces résultats indiquent que le chlore est capable de désinfecter les eaux et de fournir un résiduel de désinfection dans ces eaux pour prémunir contre les contaminations occasionnelles. Dans le cadre de l'étude de la cinétique de la désinfection au chlore Hassen Abdennaceur et al., (2000) ont montré que la réaction du chlore avec l'Ammonium est très rapide de sorte que la transformation du chlore en chloramines s'effectue en moins d'une minute. Ils ont par ailleurs trouvé que la dose de chlore influence le plus la cinétique de l'inactivation des coliformes fécaux et des streptocoques fécaux (Hassen Abdennaceur et al., 2000).

Tableau 11 : nombre de germes en fonction de la dose et des formes de chlore

\begin{tabular}{lllllll}
\hline Dose de $\mathrm{Cl}_{2}$ ou formes de $\mathrm{Cl}_{2}$ & \multicolumn{3}{l}{ Nombre de germes totaux (ufc) } & \multicolumn{3}{l}{ Nombre de coliformes totaux (ufc) } \\
\cline { 2 - 7 } & $\mathrm{P} 1$ & $\mathrm{P} 10$ & $\mathrm{P} 11$ & $\mathrm{P} 1$ & $\mathrm{P} 10$ & $\mathrm{P} 11$ \\
Témoin sans chlore (F1) & 9500 & 310 & 23 & 10 & 6 & 3 \\
Moitié chloramines (F2) & 400 & 120 & 15 & 4 & 2 & 0 \\
Maximum des chloramines (F3) & 110 & 20 & 10 & 0 & 0 & 0 \\
Break point (F4) & 81 & 10 & 8 & 0 & 0 & 0 \\
$25 \%$ au-delà du break point (F5) & 81 & 10 & 5 & 0 & 0 & 0 \\
\hline
\end{tabular}




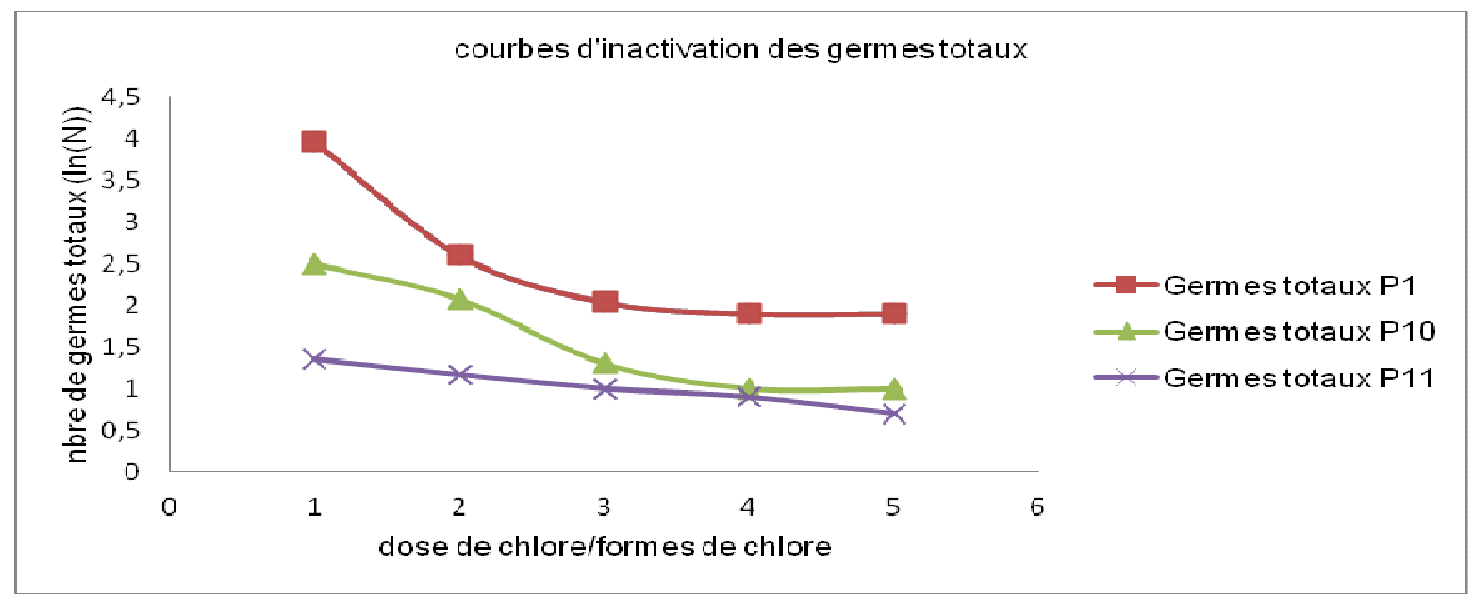

Figure 2 : courbes d'inactivation des germes totaux des eaux de trois puits

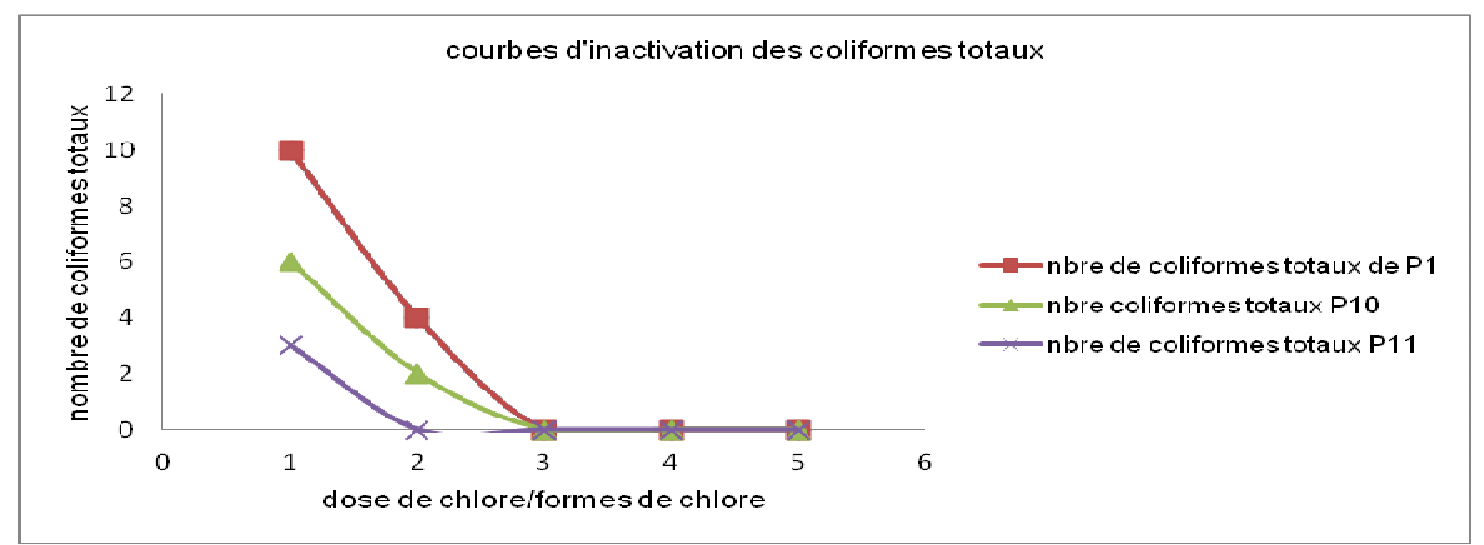

Figure 3 : courbes d'inactivation des coliformes totaux des eaux de trois puits

\section{CONCLUSION}

Aux termes de notre étude sur les eaux de puits et de forage dans deux localités du grand Lomé, il ressort que la qualité physicochimique et bactériologique des eaux des puits étudiés n'est pas conforme aux normes OMS donc impropre à la consommation. Ces eaux nécessitent un traitement avant leur consommation. Ainsi les études de désinfections au chlore ont été réalisées. Le potentiel de consommation en chlore de ces eaux est considérable et variable au cours du temps et représente environ $90 \%$ de la dose introduite pour des valeurs inférieures ou égales à la dose de break point. Cette consommation est corrélable aux caractéristiques physicochimiques

\section{RÉFÉRENCES BIBLIOGRAPHIQUES}

AFNOR, in Afnor (Eds) Recueil des normes françaises, Paris, 1986. Rodier J et col. 2009, l'Analyse de l'eau, eaux naturelles, eaux résiduaires et eaux de mer, 9 e édition principalement à la teneur en matières oxydables. En ce qui concerne l'incidence de la demande en chlore et de la chloration sur la désinfection de nos eaux, les résultats obtenus montrent que la mesure de la demande en chlore est indispensable au moment de la chloration. Les résultats que nous avons obtenus montrent que le rendement d'élimination est total pour les coliformes totaux et en moyenne plus de $91 \%$ pour les germes totaux. Ces échantillons après chloration présentent une bonne qualité bactériologique avec un chlore résiduel pour prémunir des contaminations occasionnelles. 
Techniques des eaux, Spécialité Hydrobiologie souterraine, Faculté des Sciences Semlalia Marrakech, Université Cadi Ayyad, 207P.

Bawa L.M., Tchakala I., Djanéyé-Boundjou G., 2008, Détermination de la demande en chlore des eaux de puits et de forages d'un quartier périurbain de la ville de Lomé : incidence sur la désinfection Journal des Sciences et Technologie -2008 Vol. $7 n^{\circ} 2$ pp. 19-24

Catherine Juery, (2012), Définitions des caractéristiques techniques de fonctionnement et domaines d'emploi des appareils de désinfection, document technique du Fonds National pour le Développement des Adductions d'Eau $\mathrm{N}^{\circ} 2$ (FNDAE 02), Ministère de l'Agriculture, de I'Alimentation, de la Pêche et des Affaires Rurales, 58P

Derwich E., Benaabidate L., Zian A., Sadki O. et Belghity D., 2010, caractérisation physicochimique des eaux de la nappe alluviale du haut Sebou en aval de sa confluence avec oued Fès. Larhyss Journal, ISSN $1112-3680, n^{\circ} 08$, pp. 101-112.

FAMBI Komlan, 2009, Évaluation de la contamination des eaux de puits des quartiers centraux de la commune de Sokodé (Togo), mémoire du diplôme d'ingénieur des travaux en Gestion de l'eau et de l'Environnement, École Supérieure des Techniques Biologiques et alimentaires (ESTBA), université de Lomé.51p.

Fondation de l'Eau Potable Sûre (FEPS), 2007, La chloration, www.safewater.org

Guergazi S. et Achour S., 2011, effet de la désinfection par le chlore sur la pollution des eaux de surface de la région de Biskra. Larhyss Journal, ISSN 1112-3680, nº9, Décembre 2011, pp. 131-138

H. Shayeb, T. Riabi, M. Roustan et A. Hassan, 1998, Étude expérimentale et modélisation de la désinfection par le chlore des eaux usées épurées. Revues des Sciences de l'eau, 4 (1998), pp517-536.

H. Taouil, S. Ibn Ahmed, A. El Assyry, N. Hajjaji et A. Srhiri, 2013, Physicochimie de l'eau des puits Tyikomiyne, région de Talssint (Maroc Oriental) ScienceLib Editions Mersenne : Volume 5, $\mathrm{N}^{\circ}$ 13051, ISSN 2111-4706.

Hahn H. J., 2006, The GW-Fauna-Index: A first approch to a quantitative ecological assessment of groundwater habitats. Limnologica 36: 119-137.
Hassen Abdennaceur; Heyouni Abderrahim; Shayeb, Hedi; Cherif Mohamed; Boudabous, Abdellatif, 2000, inactivation of indicator bacteria in wastewater by chlorine a kinetics study. Bioresource technology.

Hawa SAMAKE, 2002, "analyse physico-chimique et bactériologique au L.N.S. des eaux de consommation de la ville de Bamako durant la période 2000 et 2001 "thèse de Doctorat d'état en Pharmacie (Diplôme d'État) 77P.

Injection Technical Control (ITC), 2007, CHLORATION $D E$

POTABLE,www.itc.es/pdf/Technical documents IAgua-marca-Fra.pdf.

M. Makoutode, A.K. Assani, E-M. Ouendo, V. D. Agueh et A. Diallo, 1999, Qualité et mode de gestion de l'eau de puits en milieu rural au Benin : cas de la sous-préfecture de Grand-Popo. Médecine d'Afrique Noire : 1999, 46 (11).

MEYBECK M., FRIEDRICH G., THOMAS R., CHAPMAN D. (1996), Rivers Water quality assessments: a guide to the use of biota, sediments and water in environment monitoring, Chapman edition, 2nd ed. E \& FN Spon, London, pp. 59-126.

Organisation Mondiale de la Santé (OMS), Genève 2004, directives de qualité pour l'eau de boisson troisième édition, Volume 1 Recommandations.

REJSEK F. (2002), Analyse des eaux. Aspects réglementaires et techniques, Série Sciences et techniques de 'environnement, Centre régional de documentation pédagogique d'Aquitaine, Bordeaux, France.

Saâdia BRICHA, Khadija OUNINE, Saïd Oulkheir, Nourredine EL HALOUI, et Benaissa ATTARASSI, 2007, Étude de la qualité physicochimique et bactériologique de la nappe phréatique M'nasra (Maroc). Afrique SCIENCE 03(3) (2007) $391-404$.

Séby Paul, 2006, Étude de la chloration sur le réseau d'eau potable du syndicat d'Annonay - Serrières (07) mémoire de stage Licence professionnelle "Protection de l'environnement " Option Gestion des Eaux Urbaines et Rurales, 50p.

Wassila HAMDI, 2011, Qualité hygiénique et caractéristiques physicochimiques des eaux domestiques de quelques localités de la cuvette de Ouargla, Magister en Biologie Option : Microbiologie appliquée, Université Kasdi Merbah - Ouargla, 88p. 